\title{
O papel do Social-Commerce nas Intenções de Comportamento do Consumidor
}

\author{
Kennedy Jorge CANUTO \\ Universidade Federal de Campina Grande (UFCG), Campina Grande, PB, Brasil \\ Edvan Cruz AGUIAR \\ Universidade Federal de Campina Grande (UFCG), Campina Grande, PB, Brasil \\ Manoela Costa POLICARPO \\ Universidade Federal de Campina Grande (UFCG), Campina Grande, PB, Brasil
}

Received 23 dez. 18; Accepted 22 jan. 19

Evaluation System: Double Blid-Review

Editor: Jose Lindenberg Julião Xavier Filho, Dr.

ISSN: $2594-8040$

To cite this paper: Canuto, K. J., Aguiar, E. C., \& Policarpo, M. C. (2018). O papel do Social-commerce nas Intenções de

Comportamento do Consumidor. Journal of Perspectives in Management - JPM, 2(2), p. 34-50.

\section{Resumo}

Este artigo objetivou analisar o papel do social-commerce nas intenções de comportamento do consumidor em sites de compras coletivas. Por meio de uma abordagem quantitativa de caráter descritivo, fazendo uso de correlação e regressão, aplicou-se um survey online junto ao público-alvo (consumidores de sites de compras coletivas), obtendo uma amostra de 210 respondentes. Os resultados evidenciam que a sensação de presença social, os comentários dos outros consumidores e a familiaridade com o site se relacionam positivamente com a confiança do consumidor para com o site. Ademais, verificou-se que consumidores sentem mais confiança quando eles avaliam os comentários de terceiros, levando às intenções de compra. O estudo evidencia a importância do social-commerce nas intenções de comportamento do consumidor em sites de compras coletivas.

Palavras-Chave: Social-Commerce, Comportamento do consumidor, Sites de compra coletivas, Confiança. 


\section{Introdução}

O consumidor contemporâneo tem tendência em buscar por facilidades e comodidades e sempre que possível prefere comprar produtos sem precisar ir aos ambientes do varejo físico. Para isso, as redes socias são bastante utilizadas já que correspondem a espaços virtuais em que várias pessoas ou empresas se relacionam por meio de troca de mensagens e compartilhamento de conteúdo. Dessa forma, se constitui uma interação entre os usuários facilitando dessa forma a compra e venda de diversos produtos e serviços através de novas tecnologias e aplicações da internet.

Web 2.0 é o termo concebido pela empresa americana O'Reilly Media, para identificar as tecnologias e aplicações avançadas da Internet como blogs, Wikipédia, tecnologia da informação e redes sociais. Ela representa uma nova geração de serviços online pois aprimora as formas de publicação, organiza e compartilha informações; além de aumentar a comunicação entre os usuários. Enquanto plataforma, disponibiliza novas estratégias mercadológicas e processos de comunicação "potencializando" as "possibilidades" de trabalho coletivo e participativo, com a construção, disseminação e compartilhamento de informações apoiadas e intermediadas pelo computador (O'Reilly, 2005). Sendo assim, um importante aliado de lojas de comercio eletrônico, dando suporte ao marketing e as vendas.

Em função da proliferação da Web 2.0, o e-commerce evoluiu para o que se entende por socialcommerce (Ahmad \& Laroche, 2017). O social-commerce é o uso da aplicação da web 2.0 (Facebook, Twitter, Youtube, Blogs, etc.) para apoiar a intenção de pessoas em um contexto onde a contribuição dos usuários pode ajudar na aquisição de produtos ou serviços online (Liang \& Turban, 2011). De modo que se configura como um novo desenvolvimento do e-commerce gerado pelo uso das mídias sociais para melhorar as experiencias de compra online dos clientes, trazendo diversas regalias para os consumidores e varejistas.]

O social-commerce vem crescendo principalmente com a utilização do marketing através de canais digitais, troca e compartilhamento de informações e de redes sociais que buscar atrair mais clientes e mudar o comportamento do consumidor através de propagandas, incentivos e promoções. As redes sociais permitem às empresas interagir com milhões de clientes ao mesmo tempo sem investir uma fortuna em marketing para utilizar as mídias sociais (Gamboa \& Gonçalves, 2014). Isso às tornam bastante atrativas pela facilidade de uso e do seu grande alcance através de anúncios rápidos que atraem a atenção dos consumidores que os levam a tomadas de decisões.

Diversas vantagens, benefícios e oportunidades podem ser adquiridos no contexto da web 2.0 e do social-commerce. Tanto para as empresas por meio dos baixos custos de implementação e de manutenção, além de serem muito flexíveis e também para os consumidores que através da interação com outros usuários, tem acesso a informações atualizadas para pesquisa e comparação dos produtos e serviços desejados. Devido à facilidade de acesso, através de dispositivos móveis (celulares) e fixos (computadores), de modo que, os usuários se comuniquem através de relações socias e interajam entre si.

Comunidades online e fóruns da Internet são ambientes que facilitam a interação social dos indivíduos. A interação social pode ser definida como qualquer ação na qual um indivíduo se engaja e que afeta outros consumidores, avaliações ou decisões relativas a um produto ou serviço (Godes \& Mayzlin, 2004). Por meio da interação social nas redes sociais torna-se possível que os consumidores que realizam atividades de relações sociais fiquem mais propensos a seguir com a intenção de fazer uma compra (Liang \& Turban, 2011, 2011; Park, Shin, \& Ju, 2014). Formando-se redes e mídias sociais para dar suporte a estas interações.

Verifica-se que o social-commerce ocorre por meio dos consumidores que utilizam as mídias sociais em rede com outros usuários, expressando seus gostos, avaliações, recomendações e experiencias de compra online, a diferentes produtos ou serviços (Chen \& Shen, 2015; Liang \& Turban, 2011). Os exemplos incluem redes sociais, blogs, Wikipédia, youtube, facebook, dentre outros sites que consistem em comunidades de clientes com interesses semelhantes de compra online, que buscam uma interação entre os consumidores através de redes sociais.

De acordo com Liang e Turban (2011), as redes sociais facilitam a aquisição de produtos através do apoio das interações dos usuários e contribuições. A utilização das redes sociais para interagir com os clientes, seja por meio de disponibilização de classificação e comentários de produtos ou compartilhamento de compras online trazem benefícios tanto para a organização como para o consumidor, uma vez que esta 
prática incentiva o comércio eletrônico impulsionando as vendas e os consumidores obtém as informações necessárias para aquisição de um produto ou serviço desejado através de um redes e aplicativos de mídia social.

De acordo com Huang e Benyoucef (2015), o desenvolvimento de aplicativos de mídia social tem dado origem a um novo paradigma do e-commerce chamado social-commerce. Os recursos sociais inerentes a essas aplicações promovem a interação do usuário e contribuições que facilitam a compra e venda online de produtos e serviços. Segundo o mesmo autor o social-commerce pode ser obtido de duas formas: nas plataformas de mídia social, e mídia social para a através dos sites de e-commerce. Logo, uma ferramenta indispensável para empresas que desejam investir no comercio eletrônico e obter sucesso e para consumidores que desejam obter informações sobre o produto ou serviço desejado. Desse modo, entende-se que o social-commerce pode afetar no comportamento dos consumidores no ambiente de comercio eletrônico.

Alguns estudos já investigaram o papel do social-commerce e suas implicações no comportamento do consumidor, por exemplo, Ganesh, Reynolds, Luckett e Pomirleanu (2010), "Motivações de compras online, e e-Store Atributos: Uma Análise do comportamento de Compras Online Patrocínio e tipologias" realizaram um estudo que aborda algumas questões relativas à forma de como os compradores online se compararam com os compradores de varejo tradicional, realizado através da investigação qualitativa para identificar as atitudes dos consumidores online e obteve como resultado que há mais semelhanças do que diferenças entre consumidores tradicionais e online.

Pesquisas anteriores realizadas em um contexto de social-commerce como o estudo de Zhang, Lu, Gupta e Zhao (2014), "O que motiva os clientes a participar do comércio social? O impacto de ambientes tecnológicos e experiências virtuais de clientes" que buscou como objetivo fornecer os conhecimentos iniciais sobre a dinâmica da participação do cliente no comércio social e teve como resultado significativo que indicaram que a intenção do comércio social é determinada pelo apoio social, presença social e experiências de fluxo.

Essas experiências, por sua vez, são influenciadas pelas características percebidas de interatividade, personalização e sociabilidade. Também pode-se dizer que o social-commerce promove interações dos consumidores na internet para a comercialização de um produto ou serviço.

Em busca por realizar uma extensa revisão da literatura sobre o comportamento do consumidor no comércio social. O artigo de Zhang e Benyoucef (2016) "Comportamento do consumidor no comércio social: uma revisão de literatura" os resultados demostram um quadro explicativo com o modelo de cinco estágios do processo de decisão do consumidor, para a compreensão do comportamento do consumidor.

Portanto, segundo o estudo de Lal (2017), "Analisando determinantes que influenciam a intenção de um indivíduo para usar um site de comércio social" que teve como objetivo identificar fatores que influenciam a decisão do indivíduo de usar sites de comércio social em um contexto indiano e os resultados mostraram que existe uma relação positiva entre todos os seis fatores e um indivíduo tem a intenção de usar o site de comércio social. Além disso, o estudo identificou o apoio informativo como o fator mais importante que influencia o indivíduo tem a intenção de compra em um site de comércio social, seguido pela confiança dos membros do serviço.

No contexto brasileiro ainda são poucos os estudos que analisam as atitudes e comportamento do consumidor diante do social-commerce, assim como quais os construtos que os influenciam a ter intenções de compras e tomadas de decisões. Portanto, neste sentido, o presente artigo propõe analisar qual o papel do social-commerce nas intenções de comportamento do consumidor em sites de compras coletivas.

\section{Fundamentação Teórica}

\subsection{Social-Commerce: Conceito e Implicações no Varejo}

Nos últimos anos a informatização levou a sociedade a transformações não imaginadas anteriormente. A criação da internet possibilitou mudanças de paradigmas tanto no quesito espaço-tempo, quanto nos seus usos sociais e comerciais. Com isso devemos observar a transformação das relações 
comerciais, visto que o comércio eletrônico atualmente movimenta transações financeiras significantes através de novas tecnologias como o social-commerce que realiza translações financeiras com o auxílio de interações dos usuários.

O social-commerce é definido como "intercâmbio de atividades relacionadas que ocorrem em, ou são influenciados por uma rede social do indivíduo mediada por computador em ambientes sociais, em que as atividades correspondem à necessidade do reconhecimento, pré-compra, compra e pós-compra fases de um ponto de troca" (Yadav, Valck, Hennig-Thurau, Hoffman, \& Spann, 2013). O social-commerce se refere ao uso das mídias sociais para as transações comerciais e atividades que são conduzidas principalmente por interações sociais e as contribuições do usuário.

Ela é um novo desenvolvimento do e-commerce gerado pelo uso de mídias sociais para envolver os clientes a interagir com outras comunidades. Sendo assim um subconjunto de e-commerce que utiliza as interações sociais e contribuições do usuário online para facilitar a compra e venda de diversos produtos e serviços. Social-commerce é diferente de e-commerce, principalmente de duas formas. Primeiramente é construído sobre a plataforma de tecnologia social. E segundo, incentiva as mídias sociais apoiadas a atividades comerciais, diferentemente do e-commerce que depende de um sistema online características relacionadas à interface de usuário, carrinho de compras, motor de busca ou sistemas de recomendação baseados na preferência que são usadas para influenciar o comportamento de compra online (Chen \& Shen, 2015). Combinando desta forma, o conteúdo gerado pelo utilizador com funcionalidades de rede social que são usados para criar, iniciar, e de circulação de informações em redes sociais online.

Em um sentido amplo, o social-commerce envolve o uso de mídia baseados na internet que permitem que as pessoas a participar no marketing, venda, comparando, curadoria, compra e compartilhamento de produtos e serviços tanto em online e offline, mercados e comunidades. Ela não é apenas uma simples fusão entre e-commerce e redes sociais tecnologia, são independentemente de assumir a forma de adicionar uma camada social para e-commerce web sites ou ligar uma camada de varejo em sites de mídia social (Zhou, Zhang, \& Zimmermann, 2013). De forma que, as organizações através do uso dessas mídias têm um contato maior com os consumidores.

Concedendo, desse modo, novas oportunidades para as organizações se engajarem com os consumidores online diversão utilizando diferentes plataformas de mídia social caracterizado como sendo vivo, envolvente, interativa. Em que os consumidores podem fazer sua compra online enquanto comentam sobre suas compras, recebem recomendações de amigos ou têm feedback das empresas. Aumentando as relações entre marca e consumidor e criando uma comunicação mais clara entre o comerciante, o cliente e entre os próprios clientes entre si, através das mídias e redes sociais.

As mídias sociais funcionam como uma ferramenta de marketing que através delas pode-se expor os produtos, estabelecer um relacionamento com o público, conquistar mais consumidores e fazer campanhas de marketing. Mantê-las atualizadas e com conteúdo interessante é essencial para fortalecer esse vínculo, ampliando a rede de clientes, atingindo mais pessoas, aumentando a quantidade de vendas para que atenda o cliente de forma efetiva, personalizada e pessoal, fidelizar um número maior de clientes. No socialcommerce o principal objetivo do vendedor é converter os clientes em defensores da marca, já que ele permite os usuários a participar do marketing, vendas, compra e compartilhamento de produtos e serviços tanto no ambiente online como no offline (Hajli \& Featherman, 2017). Para que assim, possam ganhar a confiança para interagir com outros usuários a respeito de um produto ou serviço adquirido.

Os consumidores tendem a fazer questão de expressar seus gostos, desgostos, avaliações e experiências de marca. O sistema de mídia social é o meio preferido que as marcas usam para interagir com os consumidores, enquanto o consumidor está à vontade para se socializar, e aprender sobre suas atitudes e necessidades. Os recursos sociais inerentes a essas aplicações promovem a interação do usuário e a contribuição que facilitam a compra e venda online de produtos e serviços (Huang \& Benyoucef, 2015). De maneira que, através dessa interação os possam utilizar recomendações, comunidades sociais e avaliar comentários de outros usuários. 


\subsection{Dimensões do Social-commerce}

O social-commerce tem três dimensões: recomendações e encaminhamentos, fóruns e comunidades, avaliações e comentários (Hajli, 2015). Uma vez que as recomendações e encaminhamentos são ferramentas com as quais os consumidores endossam as suas experiências com produtos e serviços online. Compartilhando experiências de compra dentro de um círculo social e receber recomendações de produtos que você possa gostar de acordo com as suas últimas buscas e/ou compras são alguns exemplos.

Os fóruns e comunidades oferecem suporte à interação do usuário e os conteúdos gerados pelos utilizadores proporcionam a possibilidade de dividir experiências de compra online, o comércio social se fortalece. Fóruns ligados a plataformas de e-commerce ajudam consumidores a descobrirem, selecionarem e encaminharem produtos. São ambientes controlados por moderadores e as informações divulgadas geralmente passam por uma curadoria experiente.

As avaliações e comentários o mais conhecido "conjunto" de ferramentas sociais que permite que os usuários troquem feedbacks sobre produtos. Através das experiências pessoais, essas atividades ajudam a informar demais consumidores que possam vir a se interessar por determinado produto ou serviço.

Segundo Marsden (2010), não há nenhuma fórmula estabelecida para iniciar um social-commerce de sucesso. O autor completa: "a maioria dos e-commerces estão envolvidos com o comércio social ainda em fase experimental. Executar testes é a melhor maneira de estabelecer o que serve para cada empresa"

Marsden (2010) traz o conceito de aprendizagem social, que, segundo ele, é "a capacidade de aprender através do conhecimento e das experiências de pessoas que conhecemos e/ou confiamos" Através das dimensões do social-commerce é possível explicar do porque funcionarem e de e como atraem os consumidores a realizar uma compra em sites de compra online. E assim, entender os motivos que levam as intenções de comportamento do consumidor.

\subsection{Modelo de Análise Proposto}

Conforme mencionado anteriormente por (Hajli, 2015), o social-commerce vem se destacando no mercado online, uma vez que os clientes costumam pesquisar, interagir e observar os comentários de outros usuários. A partir dessas mudanças no comportamento de consumidor, os clientes passaram a obter uma sensação de presença social que influencia na hora da tomada de decisão.

\section{H1: A sensação de presença social no ambiente social-commerce se relaciona positivamente com a confiança do consumidor para com o site de compras coletivas.}

As marcas investem cada vez mais em tecnologias de interação com o cliente (por exemplo, sites, blogs, redes sociais) em um esforço para aumentar sua conexão e construir relacionamentos com os consumidores (Rowley, 2009). Neste sentido, o mercado online vem se adequando para essa nova realidade, mudando seus princípios básicos. Demostrando interesse na opinião do consumidor assim como na forma de atrair seus clientes utilizando as redes sociais como marketing, canais de bate papo para interagir com os clientes, dando a possibilidade de os clientes classificarem e avaliarem o produto adquirido para que outros ver e tomar uma decisão.

H2: Ler e avaliar os comentários de terceiros no ambiente social-commerce leva o consumidor a níveis positivos de confiança com relação ao site de compras coletivas.

Os sites oferecem o tradicional e-commerce (venda do produto online), mas também buscam se diferenciar dos demais para se manterem competitivas em relação aos seus concorrentes, investindo em novas tecnologias e formas de levar os clientes até ela. Huang e Benyoucef (2015) demostra que as empresas enfrentam desafios em fazer seus sites socialmente atraentes para implementar as funcionalidades que 
O papel do Social-commerce nas Intenções de Comportamento do Consumidor

abordam as necessidades dos seus clientes. Estes pontos explicam a escolha do objeto de estudo e os argumentos da pesquisa através da aplicação de questionários para o público-alvo.

\section{H3: A familiaridade com o site de compras coletivas se relaciona positivamente com a confiança do consumidor quanto sua à decisão de compra.}

Pesquisas anteriores investigaram o papel da familiaridade com uma marca ou produto na percepção dos usuários, como, por exemplo, intenção de compra (Gefen, 2000; Laroche, Kim, \& Zhou (1996). No entanto, o efeito de familiaridade com a plataforma online sobre as percepções, os clientes beneficiam-se de uma investigação mais aprofundada (Lim \& Van Der Heide, 2014). Assim, a familiaridade com uma plataforma online é o grau em que um consumidor compreende os procedimentos do site.

H4: A confiança do consumidor para com o site de compras coletivas, no ambiente social-commerce, se relaciona positivamente com as suas intenções de compra.

Alguns estudos têm examinado os efeitos da confiança em ambientes de negócios online, porque esses ambientes são imprevisiveis e não há nenhum face-a-face as interações entre clientes e vendedores (Doney \& Cannon, 1997; Gefen, 2000; Jones \& Leonard, 2008). Esta incerteza que motiva os consumidores a, cuidadosamente, avaliar todas as empresas de social-commerce. Estudos anteriores têm utilizado o termo "online" para se referir a confiança no negócio online envi-ronments. Por exemplo, Corritore, Kracher e Wiedenbeck (2003) definiram a confiança online como garantia e expectativa do consumidor, que as empresas online não abusem de características distintas dos ambientes online, para seus próprios lucros e que eles são confiáveis em cuidar dos clientes com honestidade, justiça e fidelidade. No próximo capitulo serão apresentadas as metodologias de pesquisas adotadas no estudo.

\section{Material e Métodos}

O presente capítulo apresenta os procedimentos metodológicos adotados a fim de alcançar a realização desta pesquisa. Ele está divido nas seguintes seções: Natureza da pesquisa, Procedimentos de coleta e Procedimentos de análise de dados.

\subsection{Natureza da Pesquisa}

O presente trabalho apresenta uma abordagem quantitativa de caráter descritivo, uma vez que objetiva analisar qual o papel do social-commerce nas intenções de comportamento do consumidor. De acordo com Fontelles, Simões, Farias e Fontelles, (2009), o estudo quantitativo é aquele que trabalha com variáveis expressas sob a forma de dados numéricos e emprega rígidos recursos e técnicas estatísticas para classificá-los e analisá-los, tais como a porcentagem, a média, o desvio padrão, o coeficiente de correlação e as regressões, entre outros. E segundo o mesmo autor para o estudo quantitativo se classifica como descritivo quando visa apenas a observar, registrar e descrever as características de um determinado fenômeno ocorrido em uma amostra ou população.

O estudo classifica-se como transversal, sendo elaborado com propósito de mensurar as características de uma amostra em um único período no tempo (Hair, Black, Babin, Anderson, \& Tathan, 2009). Dessa forma foi realizado um estudo por meio do método Survey, que segundo Hair, Babin, Money e Somouel (2003), é um procedimento para coleta de dados primários a partir de indivíduos. Os dados podem variar entre crenças, opiniões, atitudes e estilos de vida, até as informações gerais sobre a experiência do indivíduo. Fowler e Cosenza (2009), destaca que o objetivo de uma survey é produzir estatísticas, isto é, descrições quantitativas ou numéricas sobre alguns aspectos de determinada população. Vale ressaltar que se tratou de um levantamento online. 
Os dados foram coletados junto ao público-alvo (consumidores que realizaram compras em sites de compra coletiva nos últimos três meses). A seção seguinte detalhas os procedimentos inerentes à coleta dos dados.

\subsection{Procedimentos de Coleta}

O instrumento de coleta de dados foi estruturado em três seções, de forma que, na primeira, verificava se o consumidor tinha feito uma compra no site de compras coletiva nos últimos três meses, respondendo se Sim ou Não. Quando respondido "não", o respondente finalizava o questionário; quando respondido "sim", o respondente respondia o restante do questionário.

A segunda seção era composta por perguntas com a finalidade de descobrir qual o último site que o consumidor fez a compra, saber se eles pesquisam antes de comprar o produto ou serviço e se costumam visualizar os comentários, avaliações dos sites de compras coletiva.

A terceira e última seção foi composta por afirmações sobre as variáveis: Sensação de presença social (PS), Comentários (CC), Familiaridade (FAM), Confiança no site (CONF) e Intenção de compra (INT). O questionário online que foi aplicado através do compartilhamento e presencialmente, onde foram perguntadas todas as questões do e respondidas. As escalas utilizadas foram do tipo Likert de 7 pontos, alternando a variante para "discordo" como totalmente (1) e "concordo" como totalmente (7). O quadro 1 demostra as variáveis da pesquisa.

Foi realizada uma amostragem não probabilística por conveniência, visando à aceitação dos respondentes em participar da pesquisa. O que, conforme Kumar, Lemon e Parasuraman (2006), seria necessário para uma seleção aleatória correta. Hair et al. (2009) ressalta que, em amostras não probabilísticas, a chance de seleção de um elemento da população é desconhecida.

Quadro 1: Variáveis de interesse da pesquisa.

\begin{tabular}{|c|c|c|}
\hline Variável & Definição & Autores \\
\hline $\begin{array}{c}\text { Sensação de } \\
\text { presença social (PS) }\end{array}$ & $\begin{array}{l}\text { Avaliação geral do consumidor com relação a sua percepção de } \\
\text { interação social e sensação de presença humana, presentes em sites } \\
\text { de compras coletivas. }\end{array}$ & $\begin{array}{l}\text { Hajli, Sims, Zadeh e } \\
\text { Richard (2017). }\end{array}$ \\
\hline Comentários (CC) & $\begin{array}{l}\text { Ações em um ambiente específico dos sites de compras coletivas, } \\
\text { destinada a avaliações do usuários, dicas e discursões a respeito de } \\
\text { um produto ou serviço. }\end{array}$ & Hajli (2015). \\
\hline $\begin{array}{l}\text { Familiaridade } \\
\text { (FAM) }\end{array}$ & $\begin{array}{l}\text { Grau de conhecimento, domínio e intimidade que o consumidor } \\
\text { tem com os sites de compra coletivas. }\end{array}$ & Hajli et al. (2017). \\
\hline $\begin{array}{l}\text { Confiança no site } \\
\quad(\text { CONF })\end{array}$ & $\begin{array}{l}\text { Nível de segurança ou firmeza que o consumidor tem em sites de } \\
\text { compras coletivas. }\end{array}$ & $\begin{array}{l}\text { Hajli (2015) e Hajli et al. } \\
\text { (2017). }\end{array}$ \\
\hline $\begin{array}{l}\text { Intenção de compra } \\
\text { (INT) }\end{array}$ & $\begin{array}{l}\text { Capacidade e disposição do consumidor em realizar algum tipo de } \\
\text { compra em sites de compra coletivas. }\end{array}$ & $\begin{array}{l}\text { Hajli (2015), Hajli et al. } \\
\text { (2017) e Wang e Yu } \\
\text { (2017). }\end{array}$ \\
\hline
\end{tabular}

Fonte: Dados do pesquisador (2018).

Foram obtidas um total de 210 amostras, questionários obtidos via plataforma online, que estavam de acordo junto ao público-alvo (consumidores que realizaram compras em sites de compras coletiva nos últimos três meses), com a coleta sendo realizada durante o mês de novembro de 2018.

\subsection{Procedimentos de Análise de dados}

No que se atribui aos procedimentos de análise de dados, foram aplicados testes de normalidade das distribuições e de validação das escalas para preparação dos dados (Análise Fatorial Exploratória e 
coeficiente Alpha de Cronbach). E em seguida, a análise incluiu as estatísticas descritivas (média, mediana e desvio-padrão), para a definição da amostra e mensuração das variáveis.

Logo depois foram aplicadas estatísticas inferenciais (regressão e correlação), com objetivo de analisar a relação entre as variáveis estudadas (Hair et al., 2009; Hair, Anderson, Tathan, \& Black, 2005). Todos os dados foram analisados com base em no software (The R Project for Statistical Computing) de análise de dados e tabelados. No próximo capitulo serão apresentadas os resultados e discussões da pesquisa adotada no estudo.

\section{Resultados e Discussão}

Neste capítulo se discute e apresenta os resultados obtidos por meio da aplicação dos questionários online. Inicialmente, são feitas caracterização da amostra em percentuais e, logo após, a análise exploratória. Ademais, considera-se a caracterização da amostra e, em seguida, a mensuração das variáveis envolvidas. No final, os achados empíricos da pesquisa, junto com a análise do modelo de análise, que contribuíram para a obtenção do objetivo da pesquisa.

\subsection{Caracterização da Amostra}

Conforme mencionado anteriormente, a amostra foi composta por 210 observações (consumidores que realizaram sua última compra pela internet há três meses, no máximo). Dos quais, 208 pesquisam antes de comprar em sites de compra coletiva, logo nota-se que mais de $99 \%$ respondentes, levam em consideração pesquisar antes de tomar uma decisão de compra e a apenas 2 respondentes compram sem nenhum tipo de pesquisa. Dentre os sites em que os respondentes realizaram a compra, os resultados foram: Mercado Livre (36.2\%); Peixe Urbano (20.5\%); OLX (17.6\%); Groupon (10\%); Ali Express (2.4\%) e Outros Sites de Compras Coletivas que não tiveram indicies significativos par entra no gráfico somados obtiveram (13.3\%).

Gráfico 1: Preferência dos usuários dos sites de compras coletivas.

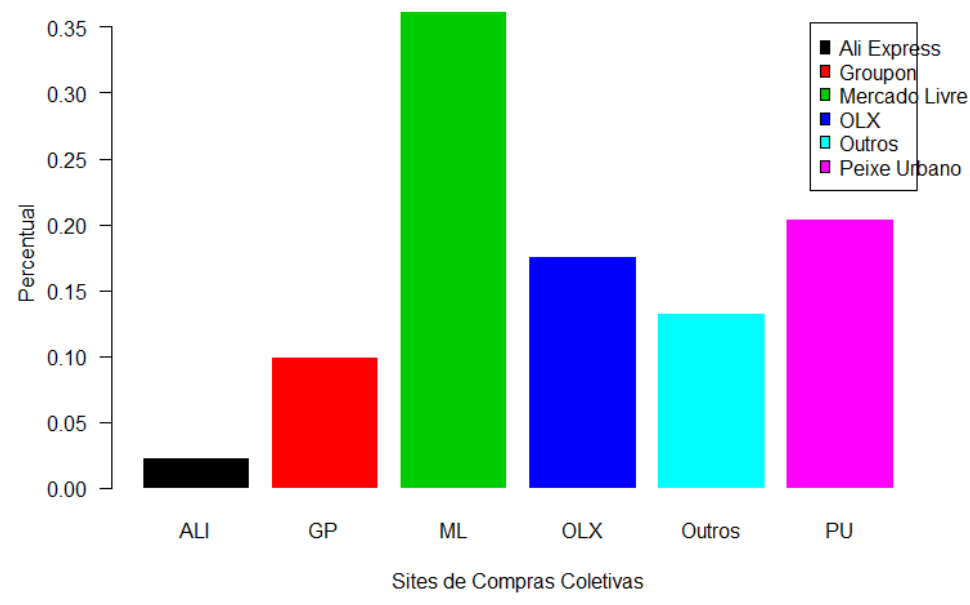

Fonte: Dados do pesquisador (2018)

O gráfico 1 demostra a preferência dos usuários dos sites de compra coletiva na hora de adquirir um produto ou serviço online. O Mercado Livre se destaca com um percentual significativo de $36,2 \%$ liderando a escolha dos clientes na hora da compra, atuando no mesmo mercado de descontos e promoções por tempo limitado aparecem o Peixe Urbano com 20,5\%, e Groupon com 10\%, a OLX acaba sendo a terceira opção na hora de decisão compra do consumidor com um percentual de 17,6\%, o Ali Express por ser um site estrangeiro ainda não é tão utilizando se comparando com os demais com um percentual de $2,4 \%$ e outros sites de compra coletiva somam $13,3 \%$ na preferência de compra do consumidor.

Verificou-se ainda que, dos 210 consumidores, 208 pesquisam antes de comprar o produto ou serviço em um site de compras coletivas, sendo mais de $99 \%$ da amostra, como também os mesmos 
costumam visualizar os comentários e avaliações dos outros consumidores nos sites de compras coletiva, antes da tomada de decisão.

O modelo de análise conforme observado na figura 1, demostra como estão relacionadas as variáveis envolvidas no estudo. Logo, observa-se que as hipóteses (H1, H2, H3) afim de descobrir o grau de relação que as três variáveis têm com a constante (Confiança). Logo, percebe-se a hipótese (H4) é a relação entre a variável (Confiança) e a constante (Intenção de compra).

Figura 1: Modelo de Análise

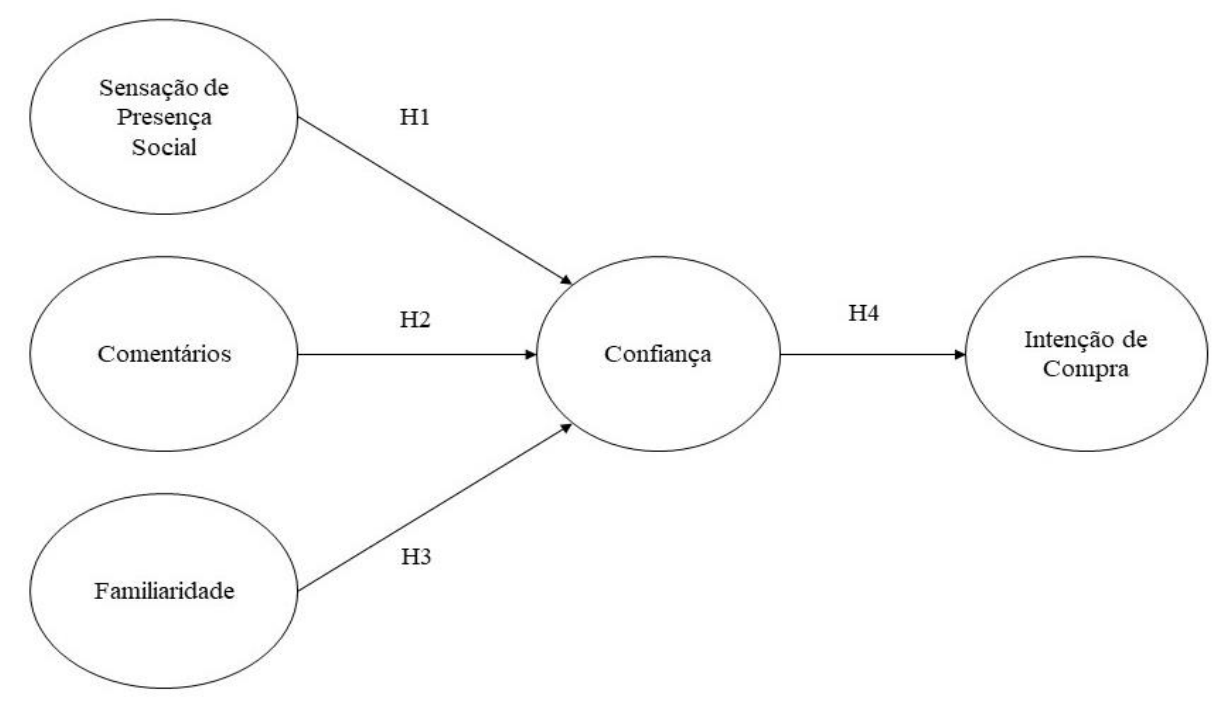

Fonte: Dados do pesquisador (2018)

No que diz respeito às estatísticas descritivas dos indicadores que formam umas variáveis latentes envolvidas no estudo, tem-se a Tabela 1.

Os resultados da Tabela 1 demostram que os consumidores de sites de compras coletiva sentem uma sensação de presença social, seja ela por comentários ou avaliações de outros usuários. Observa-se que apenas uma média dessa variável ficou abaixo de 5 com 4,97, sendo a menor média obtida no estudo. Percebe-se que a mediana da variável dos comentários foram todas 6 , o que é um ponto positivo uma vez que através dela tem-se uma proximidade e confiança no site. A variável intenção de compra obteve a maior média em relação as demais 6.05, o que demostra o desejo dos clientes em comprar em um site de compras coletiva. Vale ressaltar que, todas as variáveis analisadas obterão uma mediana acima de 5. 
Tabela 1: Variáveis Latentes

\begin{tabular}{|c|c|c|c|}
\hline Variáveis Latentes & Indicadores & Média & Mediana \\
\hline \multirow{4}{*}{ Sensação de presença social } & PS_1 & 5.26 & 6.00 \\
\hline & PS_2 & 5.25 & 5.00 \\
\hline & PS_3 & 4.97 & 5.00 \\
\hline & PS_4 & 5.24 & 5.00 \\
\hline \multirow{3}{*}{ Comentários } & $\mathrm{CC}_{-} 1$ & 5.53 & 6.00 \\
\hline & CC_2 & 5.56 & 6.00 \\
\hline & CC_3 & 5.59 & 6.00 \\
\hline \multirow{3}{*}{ Familiaridade } & FAM_1 & 5.71 & 6.00 \\
\hline & FAM_2 & 5.70 & 6.00 \\
\hline & FAM_3 & 5.67 & 6.00 \\
\hline \multirow{3}{*}{ Confiança no Site } & $\mathrm{CONF}_{-1}$ & 5.34 & 5.00 \\
\hline & CONF_2 & 5.60 & 6.00 \\
\hline & CONF_3 & 5.68 & 6.00 \\
\hline \multirow{3}{*}{ Intenção de Compra } & INT_1 & 5.57 & 5.00 \\
\hline & INT_3 & 5.30 & 5.00 \\
\hline & INT_3 & 6.05 & 6.00 \\
\hline
\end{tabular}

Fonte: Dados do pesquisador (2018)

\subsection{Caracterização da Amostra}

Antes da aplicação dos testes estatísticos de caráter inferencial, a fim de alcançar o objetivo deste trabalho, realizou-se a Análise Fatorial Exploratória que pode ser definida como um conjunto de técnicas multivariadas que tem o objetivo de encontrar a estrutura subjacente em uma matriz de dados, determinando o número e a natureza das variáveis latentes (fatores) que melhor representam um conjunto de outras observadas (Brown, 2006). E verificou-se o coeficiente de confiabilidade das escalas (Alfa de Cronbach), que foi descrito em 1951 por Lee J. Cronbach (Cronbach, 1951). É um índice utilizado para medir a confiabilidade do tipo consistência interna de uma escala, ou seja, para avaliar a magnitude em que os itens de um instrumento estão correlacionados (Cortina, 1993).

No caso da AFE, os coeficientes foram adequados, indicando 6 dimensões (as seis variáveis compostas): O método de rotação ortogonal das variáveis, VARIMAX que permitiu a identificação dos fatores. O KMO foi de 0.850 e o Teste de Esfericidade de Bartlett 2107.112, com 171 graus de liberdade a um nível de significância de 0.001 com variância total explicada acima de 60\% (mínimo aceitável).

Estes resultados confirmam a estrutura dos fatores. O próximo passo foi averiguar a consistência interna dos construtos. A Tabela 2 apresenta os coeficientes Alpha dos construtos.

A tabela 2 demostra que todos os indicadores estão acima do mínimo exigido para a validação Alfa= 0.700. Logo, as escalas de mensuração se mostram confiáveis. A partir da análise da tabela 2, percebe-se que os indicadores se mostraram adequados, já que todos os coeficientes ficaram acima do mínimo aceitável (0.7). Uma vez que a literatura sugere que acima de 0.7 os valores são satisfatórios (Malhotra, 2006). Logo, as escalas de mensuração se mostram confiáveis. Uma vez verificada a adequação das escalas, o passo seguinte correspondeu às análises que permitiram analisar o papel do social-commerce nas intenções de comportamento do consumidor. 
Tabela 2: T (X) - Cronbach's Alpha.

\begin{tabular}{c|c} 
Variável & Alfa de Cronbach \\
\hline Sensação de presença social & 0.900 \\
\hline Comentários & 0.710 \\
\hline Familiaridade & 0.770 \\
\hline Confiança no site & 0.780 \\
\hline Intenção de compra & 0.750 \\
\hline
\end{tabular}

Fonte: Dados do pesquisador (2018)

\subsection{Achados Empíricos}

Uma vez verificada a consistência das escalas foram geradas as variáveis compostas (Sensação de presença social, Comentários, Familiaridade, Confiança no site e Intenção de compra). A tabela 3 apresentam as estatísticas descritivas referentes às variáveis envolvidas no modelo.

Tabela 3: Estatísticas descritivas das variáveis

\begin{tabular}{c|c|c|c}
\hline Variável & Média & Mediana & Desvio-padrão \\
\hline Sensação de presença social & 5.18 & 5.50 & 1.21 \\
\hline Comentários & 5.55 & 5.66 & 0.72 \\
\hline Familiaridade & 5.69 & 5.66 & 0.67 \\
\hline Confiança no site & 5.54 & 5.66 & 0.86 \\
\hline Intenção de compra & 5.64 & 5.66 & 0.78 \\
\hline
\end{tabular}

Fonte: Dados do pesquisador (2018).

De acordo com a tabela 3, pode-se perceber um nível elevado nas médias dos consumidores com relação a sensação de presença social, comentários, familiaridade, confiança no site e intenção de compra. Sendo assim, os clientes de sites de compras coletivas estão satisfeitos com os sites de compra coletivas. A variável familiaridade alcançou a maior média (5.69) e o menor desvio padrão (0.67). Resultado esses semelhantes com as outras variáveis, que obtiveram média acima de (5). Vale se ressaltar que as variáveis: Comentários, Familiaridade, Confiança no site e Intenção de compra obtiveram a mesma mediana em (5.66).

Contudo a variável sensação de presença social obteve a menor média (5.18) e o maior desvio padrão de (1.21), mas a sua mediana foi de 5.50, o que demostra que ela tem um nível de similaridade semelhante em relação as demais variáveis.

\subsubsection{Análise de Correlação das Variaveis}

Neste ponto são apresentados e discutidos os resultados referentes aos testes inferenciais realizados, a fim de alcançar os objetivos da pesquisa. A princípio foi feita uma análise de correlação de forma a analisar a relação entre os construtos investigados.

Segundo Stevenson (1986) a correlação mede a força, ou grau, de relacionamento entre duas variáveis; a regressão dá uma equação que descreve o relacionamento em termos matemáticos. O autor ainda cita que a regressão compreende a análise de dados amostrais para saber se e como duas ou mais variáveis estão relacionadas umas com a outra numa população e, tem como resultado uma equação matemática que descreve o relacionamento. $\mathrm{O}$ coeficiente de correlação pode variar em termos de valor de $-1 \mathrm{a}+1$. Caso o valor da correção for igual a 0 , significa que não existe correlação e quanto maior for o valor absoluto do coeficiente, mais forte é a relação entre as variáveis. 
Logo, objetivou-se investigar a existência de relações entre a similaridade e as demais variáveis analisadas. A figura 2 apresenta os coeficientes de correlação e os indicativos da significância estatística entre as variáveis ( $\mathrm{p}$-valor $\leq 0.05)$.

Figura 2: Coeficientes de Correlação.

\begin{tabular}{|c|c|c|c|c|}
\hline Sociall & $0.57^{* *}$ & $0.36^{* * *}$ & $0.67^{* *}$ & 0.21 ** \\
\hline 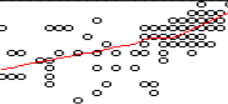 & Commp哩 & $0.60^{* *}$ & $0.70^{* *}$ & $0.51^{* *}$ \\
\hline & 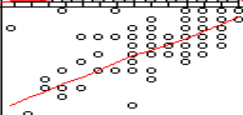 & Familifïr & $0.55^{* *}$ & $0.69^{* *}$ \\
\hline & 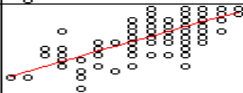 & 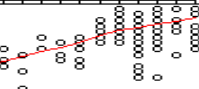 & & $0.36^{* * *}$ \\
\hline & 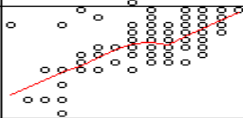 & & & Intente \\
\hline
\end{tabular}

Fonte: Dados do pesquisador (2018).

Conforme pode ser observado na figura 2, observa-se que todas as correlações envolvidas no estudo apresentaram se positivas. Além disso, as correlações se mostraram-se estatisticamente significativas (pvalor $<0.05$ ) entre as variáveis.

Dessa forma, analisando os coeficientes de relação, e observando as variáveis familiaridade e intenção de compra $(0,69)$, percebe-se que quando mais familiaridade e conhecimento o cliente tiver com o site, mais chances ele vai ter de tomada de decisão de compra no site. Essa relação impacta nas atitudes do cliente perante o varejista. Esse resultado se relaciona com os resultados de Van Der Heide e Lim (2016) como informou que os usuários familiarizados com social-commerce são mais prováveis confiar em conteúdo o qual poderia motivar a sua intenção de compra sobre plataformas de comércio social.

Como visto na matriz de correlação gráfica, a relação entre sensação de presença social e comentários tem um coeficiente forte e estritamente significativo (0.57). Desse modo, percebe-se que ao observarem os comentários em sites de compra coletiva, tem-se um aumento na interação social entre os clientes. Assim, verifica-se que ferramentas interacionais diferentes em sites de comércio social aumentam a sensação de presença social, como fotos, comentários, gostos e emoticons (Naylor, Lamberton, \& West, 2012).

Outro resultado que vale ressaltar é a correlação estritamente significativa e positiva entre sensação de presença social e intenção de compra $(0,21)$, mesmo sendo o menor valor de correlação da figura (2) e uma correlação baixa, essa correlação é positiva, com isso, consta-se que através da capacidade que os clientes tem em de projetar suas características pessoais na comunidade online, aumenta-se as chances de compra de outros usuários. Este resultado reflete o com o estudo de Gefen e Straub (2004) indicaram que a presença social aumenta as intenções de compra em plataformas online através da elevação da integridade, previsibilidade, capacidade e benevolência.

A correlação entre confiança e familiaridade (0.55) resulta em um coeficiente positivo e estritamente significativo. Com isso, consta-se que o cliente ao estar confiante no site, o seu nível de familiaridade com site de compras coletivas é susceptível de aumento. Isso traz confiança para a transação. Uma vez que a familiaridade com uma plataforma online é o grau em que um consumidor compreende os procedimentos do site (Gefen, Karahanna, \& Straub, 2003).

O coeficiente de correlação entre sensação de presença social e familiaridade (0.36). Desse modo, o consumidor ao perceber uma interação com os outros consumidores no ambiente, afeta o seu comportamento no que fiz respeito a intenção de se familiarizar e interagir no site. Com isso, entende-se que a sensação de 
presença social entre os consumidores pode afetar no comportamento do consumidor e ele estará mais propício a ser mais fiel ao site.

Outro resultado relevante é a correlação forte e positiva entre comentário e confiança (0.70). Assim, verifica-se que o maior valor de correlação existente na Tabela (2), é uma correlação moderada. Desse modo, percebe-se que o consumidor ao estar confiante, interfere no seu comportamento em realizar algum tipo de comentário para outros consumidores presente no ambiente. Conforme os resultados da pesquisa de Ba e Pavlou, 2002; Ono et al. (2003), demostram que para o cliente os feedbacks, comentários e avaliações, promovem um maior nível de confiança.

\subsubsection{Análise do Modelo Proposto}

Para validação do modelo conceitua, e, consequentemente, a verificação das hipóteses de pesquisa, foi aplicado analise de regressão linear. A análise de regressão linear estuda o relacionamento entre uma variável denominada de dependente, e uma ou várias variáveis independentes. Em que objetiva-se encontrar uma equação (chamada de equação de regressão, variável estatística de regressão ou modelo de regressão) que prevê de maneira melhor a variável resposta a partir de uma combinação das variáveis explicativas, ou seja, deseja-se encontrar os valores que melhor se ajustem aos dados do problema (Hair et al., 2005).

Segundo Hair et al. (2005), a análise de regressão que envolve apenas uma variável explicativa é chamada de regressão simples, enquanto a análise envolvendo duas ou mais variáveis explicativas é denominada regressão múltipla. Nesse sentido para fins de avaliação do modelo de análise foram aplicadas duas análises de regressão: a primeira múltipla (verificando as hipóteses H1, H2 e H3) e a segunda simples (H4).

Tabela 4: Modelo 1 - Analise de Regressão

\begin{tabular}{c|c|c|c|c}
\hline Variável & $\boldsymbol{\beta}$ & S.E & T & Sig. \\
\hline Constante & 0.304 & 0.337 & 0.903 & 0.367 \\
\hline Presença Social & 0.293 & 0.037 & 7.729 & 0.001 \\
\hline Comentários & 0.432 & 0.073 & 5.904 & 0.001 \\
\hline Familiaridade & 0.231 & 0.069 & 3.351 & 0.001 \\
\hline \multicolumn{4}{c}{ Adjusted R-squared: 0.6214 } \\
F-statistic: 113.1 on 3 and 202 DF \\
\hline P-value: < 0.00000000000000022
\end{tabular}

Fonte: Dados do pesquisador (2018)

Segundo Lapponi (2000), (p-valor) é o maior valor de nível de significância para o qual o teste é significativo. Logo, conforme a Tabela (4), em todas as três situações o Sig (p-valor) está abaixo de (0.05). Dessa forma, de fato as três hipóteses foram suportadas, presença social, comentários e familiaridade se relacionam positivamente com confiança e não há sinal negativo no modelo. Inclusive o $\boldsymbol{\beta}$ (Beta) do comentário tem o maior valor (0.432), em relação a presença social (0.293) e familiaridade $(0.231)$, o que demostra que as pessoas se sentem mais confiantes quando avaliam os comentários.

De acordo com Hosmer e Lemeshov (2000) métodos de regressão têm sido importantes na análise de dados que descrevem a relação entre uma variável resposta e uma ou mais variáveis explicativas. Dessa forma, foi considerado nesse estudo, o fato do coeficiente de determinação $\left(\mathrm{R}^{2}\right)$ possuir distribuição amostral beta (Weatherburn, 1962). O Adjusted $R$-Squared $\left(\mathrm{R}^{2}\right)$ explica que neste modelo $62,14 \%$ da confiança do consumidor em um site de compra coletiva é explicado por essas três variáveis (presença social, comentários e familiaridade). Logo, o modelo é estaticamente significativo. 
Tabela 5: Modelo 2 - Analise de Regressão

\begin{tabular}{c|c|c|c|c}
\hline Variável & $\boldsymbol{\beta}$ & S.E & T & Sig. \\
\hline Constante & 3.304 & 0.330 & 11.561 & 0.001 \\
\hline Confiança & 0.328 & 0.058 & 5.579 & 0.001 \\
\hline \multicolumn{4}{|c}{ Adjusted R-squared: 0.1265} \\
F-statistic: 31.13 on 1 and 207 DF \\
P-value: 0.00000007499
\end{tabular}

Fonte: Dados do pesquisador (2018)

O estudo e a compreensão acerca do comportamento dos custos são relevantes tanto para pesquisadores quanto para profissionais, pois tal conhecimento é imprescindível à tomada de decisões gerenciais. Dessa forma, conforme pode ser observado na tabela 5 , a hipótese confiança tem relação positiva a constante intenção de compra, com o valor de o $\beta$ (Beta) em (0.328) e com $12.65 \%$ de significância em relação a constante. Logo, as hipófises foram suportadas e o modelo é estaticamente significativo com (pvalor $\leq 0.05$ ). No próximo capítulo serão apresentadas as considerações finais referentes ao estudo, de modo a demostrar as conclusões acerca da pesquisa, de suas limitações, recomendações e contribuições.

\section{Considerações Finais}

O presente trabalho investigou o papel do social-commerce nas intenções de comportamento do consumidor em ambiente online. Os resultados demostram que todas as hipóteses foram suportadas e que o modelo de análise proposto se mostra adequado, além de possuir índices significativos que constatam que a similaridade percebida que influenciam as atitudes individuais do consumidor em sites de compra coletiva.

O estudo colabora na área de pesquisa sobre o comportamento do consumidor e o impacto do socialcommerce nas tomadas de decisões. Em especial ao comercio eletrônico. Observou-se através do estudo que a presença social, os comentários e a familiaridade influenciam os consumidores a ter confiança, visto que pessoas se sentem mais confiantes quando avaliam os comentários em um ambiente online, o que leva o consumidor a ter intenções de compra e tomadas de decisões. Esses resultados fornecem suporte para a noção de que os usuários do social-commerce estão propensos a confiar.

Conforme Linda (2010), vários fatores como a qualidade da informação, comunicação e boca-a-boca podem fazer um social-commerce confiável, pois eles são criados pelos próprios consumidores. Logo, compreende-se que a sensação de presença social, familiaridade e os comentários dos consumidores geram confiança e consequentemente é um fator que influencia nas atitudes do consumidor para intenção de compra.

Constatou-se que o social-commerce é um mercado em ascensão. Conforme Zhou et al. (2013), ele tem se expandido muito na última década e seu mercado já chegou a US\$ 30 bilhões nos Estados Unidos da América. Com isso, Linda (2010) afirma que vários fatores, tais como a qualidade da informação, comunicação e boca-a-boca, podem fazer o social-commerce confiável, porque eles são criados pelos próprios consumidores. Dessa forma, se reforça a importância do estudo, visto que o social-commerce pode contribuir para a interação entre os consumidores, gerando confiança e assim, melhorando a experiencia de consumo.

Destaca-se que o trabalho apresenta algumas limitações, em primeiro lugar o seu público-alvo, por ser restrito a apenas, apenas sites de compras coletivas. Podendo se estender a os outros sites de e-commerce. Segundo o procedimento de amostragem utilizado para a pesquisa online pode ser limitante. No entanto, o questionário foi colocado em várias grandes comunidades online, simultaneamente, a fim de proporcionar um maior/maior variedade de fontes de dados e uma resposta mais representativa da amostra.

Por último, a pesquisa foi limitada a àqueles membros que realizaram uma compra em sites de compras coletivas nos últimos três meses. No entanto, os usuários do social-commerce que já realizaram uma 
compra e se lembram, também podem ser potenciais participantes. Portanto, em estudos futuros, pode-se aplicar a todos os participantes, por se tratar se um cliente em potencial.

Espera-se que o trabalho possa contribuir para realização de estudos posteriores a respeito do papel do social-commerce nas intenções de comportamento do consumidor. Um fator que pode ser estudado no ambiente do social-commerce, seria de analisar o quanto o consumidor está disposto a divulgar informações a respeito de um produto ou serviço de um site de social-commerce voluntariamente objetivando analisar o papel desse fator nas atitudes do consumidor em relação em ser um defensor da marca. Outro fator poderia ser a busca por informações, de modo que evidencie o desenvolvimento desse construto, objetivando encontrar soluções de como o consumidor encontrar o que precisa e de como, o levar até um socialcommerce.

\section{Referências}

Ahmad, S. N., \& Laroche, M. (2017). Analyzing electronic word of mouth: A social commerce construct. International Journal of Information Management, 37(3), 202-213.

Ba, S., \& Pavlou, P. A. (2002). Evidence of the effect of trust building technology in electronic markets: Price premiums and buyer behavior. MIS quarterly, 26(3), 243-268.

Brown, T. A. (2014). Confirmatory factor analysis for applied research. New York: Guilford Publications.

Chen, J., \& Shen, X. L. (2015). Consumers' decisions in social commerce context: An empirical investigation. Decision Support Systems, 79, 55-64.

Corritore, C. L., Kracher, B., \& Wiedenbeck, S. (2003). On-line trust: concepts, evolving themes, a model. International journal of human-computer studies, 58(6), 737-758.

Cortina, J. M. (1993). What is coefficient alpha? An examination of theory and applications. Journal of applied psychology, 78(1), 98.

Cronbach, L. J. (1951). Coefficient alpha and the internal structure of tests. Psychometrika, 16(3), 297-334.

Doney, P. M., \& Cannon, J. P. (1997). An examination of the nature of trust in buyer-seller relationships. The Journal of Marketing, 61(2), 35-51.

Fontelles, M. J., Simões, M. G., Farias, S. H., \& Fontelles, R. G. S. (2009). Metodologia da pesquisa científica: diretrizes para a elaboração de um protocolo de pesquisa. Revista Paraense de Medicina, 23(3), 1-8.

Fowler, F. J. Jr., \& Cosenza, C. (2009). Design and evaluation of survey questions. The SAGE handbook of applied social research methods, 375-412.

Gamboa, A. M., \& Gonçalves, H. M. (2014). Customer loyalty through social networks: Lessons from Zara on Facebook. Business Horizons, 57(6), 709-717.

Ganesh, J., Reynolds, K. E., Luckett, M., \& Pomirleanu, N. (2010). Online shopper motivations, and e-store attributes: an examination of online patronage behavior and shopper typologies. Journal of retailing, 86(1), 106-115.

Gefen, D. (2000). E-commerce: the role of familiarity and trust. Omega, 28(6), 725-737.

Gefen, D., \& Straub, D. W. (2004). Consumer trust in B2C e-Commerce and the importance of social presence: experiments in e-Products and e-Services. Omega, 32(6), 407-424.

Gefen, D., Karahanna, E., \& Straub, D. W. (2003). Trust and TAM in online shopping: An integrated model. MIS quarterly, 27(1), 51-90.

Godes, D., \& Mayzlin, D. (2004). Using Online Conversations to Measure Word of Mouth Communication. Marketing Science, 23(4).

Hajli, N. (2015). Social commerce constructs and consumer's intention to buy. International Journal of Information Management, 35(2), 183-191.

Hajli, N., \& Featherman, M. S. (2017). Social commerce and new development in e-commerce technologies. International Journal of Information Management, 37(3), 177-178.

Hajli, N., Sims, J., Zadeh, A. H., \& Richard, M. O. (2017). A social commerce investigation of the role of trust in a social networking site on purchase intentions. Journal of Business Research, 71, 133-141.

Hair, J. F., Black, W. C., Babin, B. J., Anderson, R. E., \& Tatham, R. L. (2009). Análise multivariada de dados. Bookman Editora. 
Hair, J. F. Jr., Babin, B.; Money, A. H.; Somouel, P. (2003). Essentials of business research methods. John Wiley e Sons.

Hair, J. F. Jr., Anderson, R. E., Tathan, R. L., \& Black, W. C. (2005). Análise multivariada de dados. São Paulo: Bookman.

Hosmer, D. W. Lemeshow, S. (2000). Applied Logistic Regression. John Wiley e Sons.

Huang, Z., \& Benyoucef, M. (2015). User preferences of social features on social commerce websites: An empirical study. Technological Forecasting and Social Change, 95, 57-72.

Jones, K., \& Leonard, L. N. (2008). Trust in consumer-to-consumer electronic commerce. Information \& management, 45(2), 88-95.

Kumar, V., Lemon, K. N., \& Parasuraman, A. (2006). Managing customers for value: An overview and research agenda. Journal of Service Research, 9(2), 87-94.

Lal, P. (2017). Analyzing determinants influencing an individual' s intention to use social commerce website. Future Business Journal, 3(1), 70-85.

Lapponi, J. C. (2000). Estatística Usando Excel 5 e 7. São Paulo: Lapponi, Treinamento e Editora.

Laroche, M., Kim, C., \& Zhou, L. (1996). Brand familiarity and confidence as determinants of purchase intention: An empirical test in a multiple brand context. Journal of business Research, 37(2), 115-120.

Liang, T. P., \& Turban, E. (2011). Introduction to the special issue social commerce: a research framework for social commerce. International Journal of electronic commerce, 16(2), 5-14.

Lim, Y. S., \& Van Der Heide, B. (2014). Evaluating the wisdom of strangers: The perceived credibility of online consumer reviews on Yelp. Journal of Computer-Mediated Communication, 20(1), 67-82.

Linda, S. L. A. I. (2010). Social Commerce e s-commerce em mídias sociais contexto. Unique digital.

Marsden, Paul. (2010). Social commerce: Monetizing Social Media. Unique Digital.

Malhotra, N. K. (2006). Pesquisa de marketing: uma orientação aplicada. Bookman Editora.

Naylor, R. W., Lamberton, C. P., \& West, P. M. (2012). Beyond the "like" button: The impact of mere virtual presence on brand evaluations and purchase intentions in social media settings. Journal of Marketing, 76(6), 105-120.

Ono, S., Eigenbrode, J. L., Pavlov, A. A., Kharecha, P., Rumble III, D., Kasting, J. F., \& Freeman, K. H. (2003). New insights into Archean sulfur cycle from mass-independent sulfur isotope records from the Hamersley Basin, Australia. Earth and Planetary Science Letters, 213(1-2), 15-30.

O'Reilly, T. (2005). What Is Web 2.0 - Design Patterns and Business Models for the Next Generation of Software. O'Reilly Publishing.

Park, M. S., Shin, J. K., \& Ju, Y. (2014). The effect of online social network characteristics on consumer purchasing intention of social deals. Global Economic Review, 43(1), 25-41.

Rowley, J. (2009). Online branding strategies of UK fashion retailers. Internet Res.

Stevenson, W. J. (1986). Estatística aplicada à administração. São Paulo: Harbra.

Van Der Heide, B., \& Lim, Y. S. (2016). On the conditional cueing of credibility heuristics: The case of online influence. Communication Research, 43(5), 672-693.

Wang, Y., \& Yu, C. (2017). Social interaction-based consumer decision-making model in social commerce: The role of word of mouth and observational learning. International Journal of Information Management, 37(3), 179-189.

Weatherburn, C. E. (1962). A First Course in Mathematical Statistics. Cambridge at The University Press.

Yadav, M. S., Valck, K., Hennig-Thurau, T., Hoffman, D. L., \& Spann, M. (2013). Social commerce: a contingency framework for assessing marketing potential. Journal of Interactive Marketing, 27(4), 311323.

Zhang, H., Lu, Y., Gupta, S., \& Zhao, L. (2014). What motivates customers to participate in social commerce? The impact of technological environments and virtual customer experiences. Information \& Management, 51(8), 1017-1030.

Zhang, K. Z., \& Benyoucef, M. (2016). Consumer behavior in social commerce: A literature review. Decision Support Systems, 86, 95-108.

Zhou, L., Zhang, P., \& Zimmermann, H. D. (2013). Social commerce research: An integrated view. Electronic commerce research and applications, 12(2), 61-68. 


\title{
The Role of the Social-Commerce in the Intentions of Consumer Behavior to the Retailer
}

\begin{abstract}
This article aims to analyze the role of social commerce in the intentions of consumer behavior in collective purchasing sites. Through a quantitative approach of descriptive character, using correlation and regression, an online survey was applied to the target audience (consumers of collective purchasing sites), obtaining a sample of 210 respondents. The results show that the sense of social presence, comments from other consumers, and familiarity with the site are positively related to consumer confidence in the site. In addition, it has been found that consumers feel more confident when they evaluate third-party comments, leading to purchase intentions. The study highlights the importance of social-commerce in the intentions of consumer behavior in collective purchasing sites.
\end{abstract}

Keywords: Social-commerce, Consumer Behavior, Collective buying Sites, Confidence.

\section{El papel del Social-Commerce en las intenciones de comportamiento del consumidor para el Varejo}

\section{Resumen}

Este artículo pretende analizar el papel del Social-commerce en las intenciones del comportamiento del consumidor en los sitios de compras colectivas. A través de un enfoque cuantitativo de carácter descriptivo, utilizando la correlación y la regresión, se aplicó una encuesta en línea a la audiencia objetivo (consumidores de sitios de compras colectivas), obteniendo una muestra de 210 encuestados. Los resultados muestran que el sentido de presencia social, los comentarios de otros consumidores y la familiaridad con el sitio se relacionan positivamente con la confianza del consumidor en el sitio. Además, se ha encontrado que los consumidores se sienten más seguros cuando evalúan los comentarios de terceros, lo que lleva a intenciones de compra. El estudio destaca la importancia del Social-Commerce en las intenciones del comportamiento del consumidor en los sitios de compras colectivas.

Palabras Clave: Social-commerce, Comportamiento del Consumidor, Sitios de compra colectiva, Confianza.

\section{Sobre os Autores}

\section{Kennedy Jorge CANUTO}

Graduando em Administração pela Universidade Federal de Campina Grande (UFCG)

Rua Aprígio Veloso, 882 - Universitário - Campina Grande - PB - Brasil - CEP 58429-900.

E-mail: kennedycanuto@ hotmail.com

\section{Edvan Cruz AGUIAR}

Doutor em Administração pela Universidade Federal de Pernambuco (PROPAD/UFPE). Professor Adjunto da Unidade Acadêmica de Administração e Contabilidade da Universidade Federal de Campina Grande (UAAC/UFCG)

Rua Aprígio Veloso, 882 - Universitário - Campina Grande - PB - Brasil - CEP 58429-900.

E-mail: $\underline{\text { edvan.ed@gmail.com }}$

\section{Manoela Costa POLICARPO}

Mestranda em Administração pelo Programa de Pós-Graduação em Administração da Universidade Federal de Campina Grande (PPGA/UFCG)

Rua Aprígio Veloso, 882 - Universitário - Campina Grande - PB - Brasil - CEP 58429-900.

E-mail: manoelacpolicarpo@gmail.com 